\title{
VI. On the multiple sounds and optical phœnomena produced by vibrating bodies. Theory of the bow
}

\section{M.J. Antoine}

To cite this article: M.J. Antoine (1850) VI. On the multiple sounds and optical phœnomena produced by vibrating bodies. Theory of the bow , Philosophical Magazine Series 3, 36:240, 27-40, DOI: $10.1080 / 14786445008646417$

To link to this article: http://dx.doi.org/10.1080/14786445008646417

曲 Published online: 30 Apr 2009.

Submit your article to this journal $[\pi$

ЏII Article views: 2

Q View related articles $₫$ 
On the Multiple Sounds produced by Vibrating Bodies. 27

faces in the two minerals differ. The specific gravity of the two minerals is-

Brookite.

$4 \cdot 125$ to $4 \cdot 169$ Breithaupt.

3.810 Hermann.

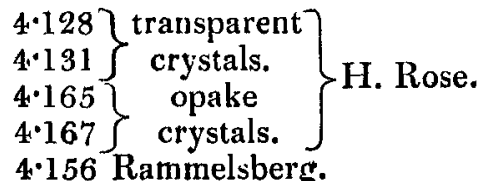

Arkansite.

$3 \cdot 854$. Shephard.

3.952 Breithaupt.

3.892

$3 \cdot 923\}$ Rammelsberg.

$3 \cdot 949$

Further, the specific gravity of Arkansite is almost identical with that of Anatase; it may therefore be said that Arkansite is native titanic acid with the form of Brookite and the density of Anatase.

SMECTITE. BY L. A. JORDAN.

[Poggendorff's Annalen, vol. Ixxvii. p. 592.]

This mineral, which occurs at Cilly in Lower Styria, and is likewise found at Zeny in Croatia, has been analysed by $L$. A. Jordan, who has found for it a composition corresponding to the formula $\left.\left.\begin{array}{l}\mathrm{Al}^{2} \mathrm{O}^{3} \\ \mathrm{Fe}^{2} \mathrm{O}^{3}\end{array}\right\} 3 \mathrm{SiO}^{3}+\underset{\mathrm{CaO}}{\mathrm{MgO}}\right\} \mathrm{SiO}^{3}+12 \mathrm{HO}$, viz.

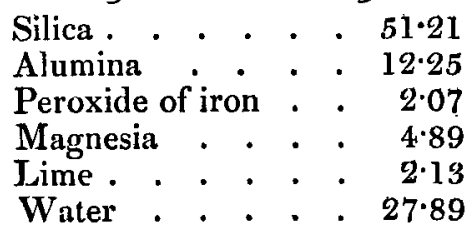

[To be continued.]

VI. On the Multiple Sounds and Optical Phanomena produced by Vibrating Bodies. Theory of the Bors. By M. J. AnTotne*.

M DUHAMEL has just published a novel and inge. N1. nious theory on the multiple sounds of sonorous bodiest. I know not what reasons have led him to imagine that justice has not been done him. On this point the learned academician is under an illusion. Physicists will render him justice, in spite of the disdain which $M$. Duhamel affects, when he supposes them as little advanced as at the period of Father Mersennus, and in spite of their well-founded belief

- From the Annales de Chimie et de Physique for October 1849.

+ See Phil. Mag, vol, xxxiv. p. 415. 
that they have long since possessed an exact, simple and direct theory of multiple sounds.

I regret that M. Duhamel has thought proper to be rery brief in transcribing the state of science, It is with fear that I undertake to be his substitule in a delicate matter, which he has studied for many years; nevertheless, I shall venture to give an historical sketch, in order better to appreciate the present position of the question.

The phænomenon of multiple sounds has been known for a very long time, for Aristotle asks by what reason the grave sounds contain also sharper sounds. Mersennus however was the frist physicist who submitted this to experimental analysis.

This ingenious observer classed the different higher sounds which accompany the fundamental note of a string, and found the same series for strings of different materials, tension and dimensions. The supplementary sounds which he could distinguish, placing himself in favourable circumstances, are the octave, the twelfth, the fifteenth, the major seventeenth, the nineteenth and the twenty-second. When accustomed to these experiments, it is easy to prolong the series beyond these limits. Mersennus classed also the tones which compose the multiple sounds of bells, as those, more curious still, which proceed sometimes simultaneously from the pipes of an organ.

In seeking the cause of this multiplicity of sounds, Mersennus made an experiment which might have led him to discover it; but the sagacity of his spirit was baffled, and the truth escaped him. He examined the vibrations of a long string, and seeing no division established, he concluded that strings do not divide to produce the simultaneous harmonics, and he therefore sought the explanation of the phænomenon in the wrong quarter.

The theory of multiple sounds did not make progress until experimental analysis had disclosed the cause of this multitude of sounds which may be separately drawn from one and the same sonorous body. It had been long known that a considerable series of successive sounds could be produced from the trumpet; Mersennus nuade the exact classification of them, and compared it to that of the different sounds which one pipe of an organ can give; but he could not discover the cause. The difficulty was great; and it was first solved, not upon aërial columns, but upon vibrating strings.

In 1673, William Noble and Thomas Pigot made that beautiful experiment, in which small pieces of paper, placed astride upon a string, are seen to remain immoveable in certain 
positions, whilst beyond they are often projected from the string. Wallis considered the fact so curious and so novel, that he gave it a place in his Treatise on Algebra. It resulted clearly from the experiments of Noble and Pigot, that the same string can separately produce the different harmonics of the fundamental tone, and that, for this, it divides itself into aliquot parts which vibrate alternately in a contrary direction, remaining separated by immoveable points or nodes.

The cause of the phænomenon being thus clearly characterized in the particular case of vibrating strings, it remained to generalize it. Daniel Bernouilli by ingenious experiments treated the case, perhaps the most difficult, of wind-instruments, and finally experiment reached by degrees all possible cases, so that it is proved that a sonorous body can produce a considerable series of very different sounds, and that for each of them it divides into a corresponding system of vibrating parts and nodal surfaces.

It would perhaps be proper now to examine the connexion which exists between multiple sounds and the cause of the different sounds which bodies can give successively. However, to suit my purpose, I shall first say a few words on the processes which physicists have devised for drawing a varied succession of isolated sounds from the same sonorous body. I shall limit myself to the case of vibrating strings, the only one which has presented notable difficulties.

When Noble and Pigot made their capital experiment, only a single regular means was known of making a string produce its harmonics successively. It was not touched, it was not directly vibrated with the bow, but the harmonic which it was desired to raise was sounded at a distance, and instantly the string began to vibrate, to form its nodes and produce the harmonic. This communication of movement by the air and the supports of the string, or, as was then said, this communication of sounds by sympathy, had been discovered by Fracaster. Mersennus had made the experimental analysis of it with care, and had found that the sounds most apt to excite by sympathy the vibrations of a string form the different harmonics of the fundamental tone; he had also remarked that the fifth, the fourth, the major third and other consonances, produce also feeble vibrations, when the string is perfect, long, and stretched on a suitable instrument. The following case is perhaps not wholly unworthy to be mentioned. Two strings are stretched on a sonometer, and they are set so near to unison as to give beats when they vibrate simultaneously. Then if one of them be made to vibrate directly, the string which has not been touched experiences very 
perceptible vibrations; and further, it causes beats to be heard. This experiment shows the imperfection of one of the processes which are indicated for tuning instruments.

In order to produce from a string the series of its harmonics in succession, Sauveur afterwards devised a second method, more convenient, and more effective than the first. As in the old process the string was not touched, the harmonic which it was desired to excite was sounded by the side, but he produced this first generating sound by vibrating the prolongation itself of the cord. The harmonics obtained in this manner are in some degree indefinite; the higher ones are not, it is true, so well characterized as the lower. Nevertheless, with a string one metre in length, Sauveur distinguished clearly thirty-two harmonics, and could hear more than a hundred and twenty-eight. Sauveur's method is, in this respect, much superior to the old process.

Sauveur did not seek whether it is possible to establish the subdivisions of the string, by vibrating it directly with the bow. This may however be attained, although the execution presents difficulties. If the bow is moved on the middle of the string, avoiding to impart the movement of totality which gives rise to the fundamental tone, we have at first only a harsh and very disagreeable grating sound. This fact, related by $\mathrm{W}$ allis as known before him, is produced, as Wallis has remarked, when the bow is drawn on one of the points of the string which correspond to its division into aliquot parts, with this circumstance however, that the sound becomes less and less disagreeable, in proportion as the divisions are more numerous.

It must not however be imagined that the phænomenon mentioned by Wallis is constantly verified. For, on passing the bow on the middle of the string and modifying its velocity and its pressure, a sharp sound of great purity is in the end obtained, after some essays. When this sound is full, it is easy to sustain it as long as is desired, even whilst increasing considerably the pressure of the bow. By this means the unm even harmonics may be drawn from a string.

It is easy to distinguish in these experiments the formation of the nodes and their position. If the string gives, for example, the nineteenth harmonic, when observing it in broad daylight, it looks as if formed of nineteen equal spindles, placed one after another. The points where these spindles unite appear immoveable; if they are lightly touched with the finger, the sound which the bow maintains is not changed, and the string is not felt to tremble. If, on the contrary, one of the spindles is touched at a proper distance from their extremities, 
the trembling of the string is felt and the sound is no longer obtained.

We may thus prove the existence of the nineteen vibrating parts, by passing the bow successively on each of them; by this manœuvre the sound will not be changed. If the bow approaches too near one of the nodes, the harmonic produced instantly disappears, and the fundamental generally supplies its place.

If it is desired to ascertain the position of the nodes, by means of light bodies placed astride upon the string, circles of paper must be used strung through the middle, or better still, a wire ring, in order that if the bow does not succeed at the first stroke in producing an harmonic, the light bodies may not be thrown off from the string.

The uneven divisions are not the onlyones which may be produced by vibrating the string directly with the bow. The even divisions are obtained by drawing the bow skilfully at a proper distance from the nodes which it is wished to form. The success of the experiment will be rendered easy by touching an instant only one of the nodes.

The method which I have just pointed oul may also serve to bring out with force, or to silence certain sounds in the concert of the harmonics which ordinarily accompany the fundamental tone.

After this perhaps too long digression, I revert to the principal question. When the cause of the successive sounds which a body can give had been proved by experiment, the explanation of multiple sounds very naturally followed,-the explanation of which so greatly puzzled Father Mersennus. It is easy, in fact, to conceive that when different modes of vibrations, capable of existing isolatedly in a body, are produced in it together, each of them gives out the sound which corresponds to it. M. Biot has developed this theory with elegance; it has since been reproduced in the works of physicists. I know not for what reasons $M$. Duhamel has not even mentioned it.

The exactitude of the commonly admitted theory on multiple sounds, is confirmed and in some sort submitted to the eye, in the phænomenon of multiple strings and rods, which is perceived when they are made to yield different sounds sinultaneously. To appreciate what there is completely demonstrative in this phænomenon, it is sufficient to bring attention to the facts.

When a rod $A B$, fig. 1 , fixed in $A$, vibrates on each side its position of equilibrium $A B$ between the extreme positions $\mathrm{AC}, \mathrm{AD}$, in a breadth which exceeds its thickness, 
two images of the rod are seen perfectly traced and as it were immoveable in the positions $\mathrm{AC}, \mathrm{AD}$. The rod passes very rapidly each intermediate position, and reaches the extreme positions $\mathrm{AC}, \mathrm{AD}$, with velocities which are annulled and then increase again by imperceptible degrees. The images produced on the retina by the rod, when it is in the positions near the minima of velocity, where it remains almost immoveable for a comparatively long time, must be more vivid than in the other positions; thence the appearance observed. The two clearly visible inages of the rod also approach by degrees, in proportion as the magnitude of the vibrations diminishes.

Fig. 1.

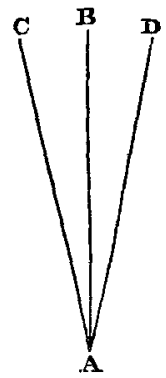

If the rod possesses at last a vibratory movement and a motion of translation, instead of two images, a very considerable number will be perceptible at regular distances over the whole extent that the movement of translation causes the rod to pass. Thus, if a knife be held in the niddle, and one of its ends be struck on a fixed obstacle, leaving the knife free to rebound and vibrate, there will be perceived ten, twenty, thirty knives regularly distributed in front of the obstacle. If an object be struck with a bow, several images of the bow will be seen, each presenting a perfect and very distinct design of all the parts of the bow.

These multiple images are easily explained. Let us suppose that a rod $A B$, fig.2,deviated towardsAC, is left to itself, and that at the same time it is impressed by a movement of translation which carries the point $A$ in the direction of the line AM. Let us Fig. 2.

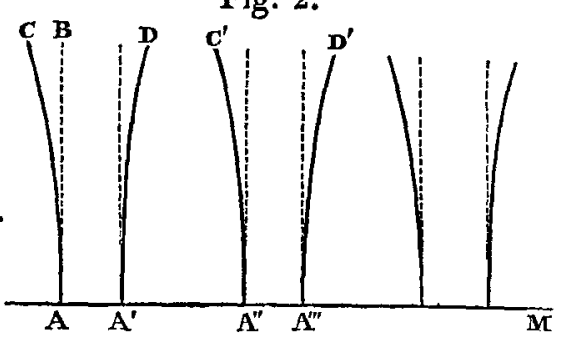
take on $A M$ the lengths $A A^{\prime}, A^{\prime} A^{\prime \prime}, A^{\prime \prime} A^{\prime \prime \prime} . .$. , traversed by the point $A$ in the time which each simple oscillation takes to accomplish. When the point $A$ is at $A^{\prime}$, the rod will be at $A^{\prime} D^{\prime}$ at the end of its first simple oscillation; in the same way the rod will terminate its second, third.... oscillation in the positions $\mathrm{A}^{\prime} \mathrm{C}^{\prime}, \mathrm{A}^{\prime \prime \prime} \mathrm{D}^{\prime} \ldots$ These positions are very near those which correspond to minima of velocity; the other positions are traversed very rapidly, and it is on this account that images of the rod are distinctly seen nearly in the positions $A C, A^{\prime} D$, $A^{\prime \prime} C^{\prime}, A^{\prime \prime \prime} D^{\prime} \ldots$ They will be seen simultaneously if the movement of translation is suitable. 
Vibrating strings present analogous phænomena.

Let us suppose that the string vibrated produces the funclamentak tone with energy, and gives the harmonics only in a slightly perceptible manner. Two very distinct images are then seen in the extreme positions $A m B$, $\mathrm{A} m^{\prime} \mathrm{B}$, fig. 3 ; these images are visible at the very places where the velocity of the string is null or extremely small, in comparison with Fig. 3.

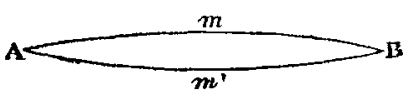
that by which it is affected when it traverses the intermediate positions.

If the bow is drawn so as only to give the octave of the fundamental sound, two equal spindles are seen placed one after the other, and each one giving two images of the string in the positions where the velocity is annulled, as in $\mathrm{A} n \mathrm{M}, \mathrm{A} n^{\prime} \mathrm{M}$, $\mathrm{M} p \mathrm{~B}, \mathbf{M} p^{\prime} \mathrm{B}$, fig. 4. These appearances are the immediate consequence of the division of the string into two distinct parts which vibrate separately.

Fig. 4.

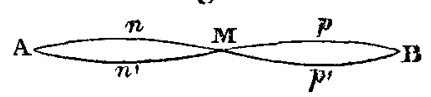

If the fundamental tone be drawn from the string and at the same time its octave, so that these two sounds are very powerful in comparison with the other harmonics, which we will suppose to be very weak, four images of the string are then seen arranged as indicated in the figure. According to the explanation commonly admitted of multiple sounds, whilst the whole string vibrates, its two halves themselves vibrate, taking contrary curves. Hence it follows, that when the string quits the position $\mathrm{AB}$, fig. 5, to take the direction toward the curve $\mathrm{A} a \mathrm{C} b \mathrm{~B}$, the firsthalf of the string $A C$ will present its concavity, for example, at $\mathrm{AB}$, and the se.

Fig. 5.

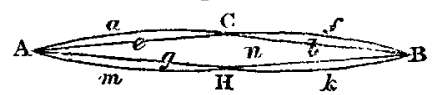
cond half, $\mathrm{CB}$, its convexity. During this transfer, the velocities of the different points of the string will tend to disappear successively: the curve $\mathrm{A} a \mathrm{C} b \mathrm{~B}$ is the spot which the different points of the string attain when their velocity becomes null.

After curving, as we have pointed out, the string will assume opposite curves, and will then return in the direction of the primitive curves, and so on. During these variations of form, the points of the string will pass from the position $\mathrm{A} a \mathrm{Ci} B$ to the position $\mathrm{A} e \mathrm{C} f \mathrm{~B}$, where their velocity will be successively annulled, then to the position $\mathrm{AgH} k \mathrm{~B}$, where the velocities are still null, then to the position $\mathrm{A} m \mathrm{H} n \mathrm{~B}$, where these velocities become null for the fourth time; and lastly, they will return to the position $\mathrm{A} \alpha \mathrm{C} b \mathrm{~B}$, where the velocities were first

Phil. Mag. S. 3. Vol. 36. No. 240. Jan. 1850. 
annulled, and where they will become null again successively for each point, if the decrease in amplitude of the vibrations is neglected.

From these considerations it follows, that during the complete vibration of the string, four distinct loci are formed in which the points of the string arrive successively without velocity, after having traversed the intermediate positions with a greater or less rapidity. Thence results the appearance of four distinct strings, curved as the lines of the figure indicate, and consequently the explanation of the optical phænomenon which is observed. It follows from these same considerations, that during an entire oscillation, the string strikes the air four different times, in passing from $\mathrm{A} a \mathrm{C} b \mathrm{~B}$ to $\mathrm{A} e \mathrm{C} f \mathrm{~B}$, from this last position to $\mathrm{Ag} \mathrm{H} k \mathrm{~B}$, then to the position $\mathrm{A} m \mathrm{H} n \mathrm{~B}$, and lastly, to the first position $\mathrm{A} a \mathrm{C} b \mathrm{~B}$. These four shocks are not identical : in the first and the third, the two halves of the string strike the air in a contrary direction, whilst in the second and the fourth, the string strikes the air in the same direction at all the points, nearly as if it moved without being divided. Thence it results that in the series of shocks which the tympanum of the ear receives, at each second shock the impression made on us must have something distinctive, and the periodical return of this phænomenon brings the simultaneous sensation of two sounds, the one the octave to the other. This is precisely the fact which observation indicates and which had to be explained.

All the preceding observations may be confirmed by completing in the following manner the experiment which we have just described.

If, by a suitable management of the bow, the octave of the fundamental tone is weakened, the spindles $\mathrm{A} a \mathrm{C} e, \mathrm{C} b \mathrm{~B} f$, $\mathrm{AgH} m, \mathrm{H} k \mathrm{~B} n$ will diminish in width, and will end in being imperceptible, when the octave has become very weak.

If, on the contrary, you weaken the fundamental sound, the spindles will persist, but the parts $\mathbf{C}$ and $\mathbf{H}$ will approach, will tend towards being confounded, and will in fact be so, when the fundamental sound is silenced.

The form of the curves will be easily determined upon which the different points of the string arrive successively without velocity, and the duration of the motion of each point in passing from one curve to the other, by aid of the formula

$$
g=\alpha \sin \frac{\pi x}{l} \sin \frac{\pi a t}{l}+\beta \sin \frac{2 \pi x}{l} \sin \frac{2 \pi a t}{l},
$$

which represents one of the possible movements of the string, and may specially represent that which we have just analysed. 
In order to demonstrate the effects of the combination of two vibratory movements on a body, we have only to produce movements sufficiently slow to enable us to follow readily their different phases. We shall easily obtain this result by the aid of a long vibrating rod, by striking this rod in a suitable manner luring its vibrations of totality, so as to produce a double vibratory movement. This experiment is not without interest.

I shall not pursue further the analysis of these different phænomena, nor shall I speak of the curious appearances presented by a vibrating string when left to itself. What I have developed suffices to render perceptible to the eyes the explanation of simultaneous harmonic sounds, as deduced from the fundamental experiments of Pigot, Noble and Sauveur.

Let us now examine the explanation of multiple sounds proposed by M. Duhamel. In this examination, we shall consider more specially vibrating strings, as offering the most elegant and interesting example of multiple sounds. To abridge and profit by the details on which we have already entered, we will suppose that the string gives simultaneously the fundamental tone and its octuve.

In the first part of his memoir, M. Duhamel establishes this proposition: "When a body is made to vibrate by several causes which separately would produce the simple sounds which it can give, its surface generally divides itself into a certain finite number of parts, in each of which the vibrations have unequal durations. These different durations have relation to sounds corresponding to the different causes, and we are in the same position as if we had several separate surfaces, each having a particular movement of vibration."

In the vibrating string which gives the fundamental tone and its octave, the middle is the only point the vibrations of which differ in duration from those of the other points; I do not find here two finite portions of string in each of which the vibrations have unequal durations. Perhaps it will be said that one of the two finite portions is reduced to a point or to the contour of the middle of the string, but then the proposition would not be new with regard to strings. Still, however, this interpretation does not appear to me admissible, for, according to the proposition, we must be in the same case as if we had several separate surfaces, each having a particular movement of vibration; thence the fundamental tone would be solely attributable to the vibrations of the middle of the string, that is to say, of a single point ; they would therefore be insensible with relation to the vibrations which give the octave. It is known that the fundamental tone may be very powerful, and D 2 
we have seen in the ordinary theory of sounds that the string produces this tone by striking the air at all its points.

M. Duhamel, whilst reproducing the preceding proposition in the same memoir, changes its sense very materially, when he says that each of the sounds exists in one or more finite parts of the surface, and appears to be perceptible there only. The isolation of each sound is therefore not absolute, but approximative; with this interpretation, the proposition of M. DLhamel allows of the string producing the fundamental tone by striking the surrounding air at all its points: only, the energy of the shocks will vary throughout the extent of the string, and may be very great in one finite part of the string, with reference to other parts. In this sense, the proposition would have little novelty.

In the second part of his memoir, M. Duhamel becomes more explicit; he abandons entirely the absolute sense of the first form of his proposition, that is to say, that which would be a real novelty, and admits, what is far from being anything new, that, during the multiple sounds of a boly, there are portions of the surface which seem to give only one sound, although however we may be sure that they give out several others. In the case of the vibrating string, that is as much as to say, if I am not mistaken, that the middle of the string does not contribute directly to the production of the octave, and that the parts adjoining this middle contribute to it only in a scarcely perceptible manner, which is evident.

In the second part of his memoir, M. Duhamel adopts fully the received ideas on the multiple sound of bodies, but he presents them under a form which is peculiar to himself, and which we proceed to examine.

He admits, as every one does, that when a string produces the fundamental tone and its octave, its vibratory movement is composed of the two movements which correspond to the separate production of these two sounds; he does not seek to prove experimentally that it is so, but he shows that if the movement of the string is compounded as we have just said, there must result from it the simultaneous sensation of the fundamental sound and its octave.

In the common theory of multiple sounds, this consequence is in some sort immediate. When the entire string makes a vibration, its two halves which vibrate at the same time make two, and thence it follows that it strikes the air four times during a complete vibration; but the shocks are not identical, they only resemble one another alternately two to two; the ear is sensible to this periodical return of such shocks, and thence the origin of the compound sensation which is perceived. 
M. Dubamel does not consider directly the shocks as they take place in reality, but he substitutes for them an equivalensystem; instead of the string affected by the compound movet ment, he substitutes two identical strings placed close to each other, each executing simple vibrations which are an octave from each other. It is certain that the movement imparted to the air, whether by the single string, or by the two equivalent strings united, is very perceptibly the same, and our organ must be affected in the same manner in the two cases; now, it is known that two adjacent strings, one of which vibrates the octave of the other, cause the two sounds to be heard simultaneously; they must therefore be thus heard by virtue of the equivalent vibrations of the single string.

If I have rightly seized the point of view which $M$. Duhamel has taken, it appears from the preceding developments, that M. Duhamel, with all the physicists of the present day, finds the origin of multiple sounds in the manner in which the vibratory movement of bodies is composed. To establish this dependence, physicists consider directly the different shocks really imparted to the air, whilst $M$. Duhamel substitutes for the body vibrating with a compound movement, an equivalent system of simple movements, the impression of which on our organs is well known.

The common theory of multiple sounds is more direct than that of $M$. Duhamel, since in it we regard phænomena as they are really produced; it is also more elementary, and consequently more simple, as it does not require us to have recourse to propositions, one of which at least is not within the reach of every one. For these two reasons, the ordinary theory appears to us preferable in general; nevertheless, in certain complicated cases, we think that the mode of demonstration proposed by M. Duhamel may have the advantage.

Whichever of the two theories be adopted, one difficulty relative to the multiple sounds of bodies remains entirely to be solved. This difficulty arises from our not having as yet been able to submit to analysis the true theory of the bow.

The bow produces four principal effects. It sustains the equality of the sounds as long as is wished; it sustains also those feeble sounds which appear to die away under the fingers of the artist, whilst the sounds given by the strings, when pinched, are at first powerful, then soon become weakened, and are very quickly lost. The bow admits of giving to sounds different degrees of strength; it serves to impart to them particular qualities by appropriately awakening the harmonics; and lastly, it can bring out separately the different harmonics from a string. By these effects, the bow is the supreme master of sounds. 
The bow acts thus by a series of slight impulses which it imparts to the string. These shocks, being continually renewed, keep up the movement which tends to become weaker; becoming slighter or more energetic, they change the amplitude of the vibrations, and consequently the strength of the sounds; by being applied to the different parts of the string, in suitable conditions, they are able to determine various modes of vibrations which give rise to the separate or simultaneous harmonics.

The impulses which the bow can impart are renewed with such great rapidity, that we cannot be assured of their existence when looking at the string. However, if the pressure of the bow is considerable and the velocity very moderate, the sounds produced present the same characters as if they were attributable to a series of shocks. Moreover a series of jerks may then be seen, which render manifest to the eye the different shocks of the bow. The experiment takes a new character of evidence when the tension of the string is very weak or the vibrations are slow.

When the experiment is performed which has just been indicated, and the jerks of the string are very perceptible, independent of the fundamental tone of the string, two supplementary sounds are heard which are the fundamental tones of the two portions of the string separated by the bow.

If the pressure of the bow be gradually diminished and its velocity increased, the jerks become more rapid, more difficult to observe, at the same time that the two supplementary sounds are weakened; as long as the supplementary sounds continue, they are as it were proofs of the shocks produced by the bow. Lastly, the jerks cease to be perceptible, and yet the two supplementary sounds are still heard, feebly it is true, but still perceptible enough to be distinguished, with a little attention. Thus the friction of the bow produces a succession of shocks, the origin of all the effects of this instrument.

It is not necessary to imagine that these shocks must be renewed in a regular manner in order to sustain the sound, for the sound of a string may be sustained by striking it gently with the finger and rapidly renewing the shocks almost in an arbitrary manner. The only difference that is remarked in the sounds sustained by the bow or by a rapid succession of shocks effected by pinching the string with the finger, is referable only to the delicacy and the lightness of the shocks of the bow.

The existence of the supplementary sounds, incontestable proofs of the shocks of the bow, is very clear and very decided, when the rapidity of the bow does not exceed certain limits. In the case where the bow moves in the ordinary way, the 
supplementary sounds become so feeble, that the perception of these sounds might be attributed to a preconceived illusion of the mind, although analogy then lends its aid to the conclusions. Nevertheless, if any doubt still remained, it would be easy to remove it by the following observations.

When the bow causes a string to sound, its hairs execute transversal vibrations. To prove this, it suffices to pass a simple wire ring around the hairs: whilst the bow moves on the sonorous string, the wire ring, by its movements, renders the vibrations of the hairs evident.

The vibrations rendered perceptible by the wire ring are very remarkable when the bow is reduced to a single hair, and especially when the place of the hairs is supplied by a sonorous cord, rubbed with resin.

Ordinarily the tension given to the hairs of the bow is such, that the sound rendered by each hair, vibrating separately, is very grave with reference to the sound which the bow should draw from a string; whatever tension is given to the hairs of the bow or to the bodies which supply their place, the transversal vibrations are always established and are constantly rendered evident by the experiment, so that the shocks of the vibrating string against the hairs, and consequently the shocks of the hairs against the string, are incontestable.

It would be difficult to say $a$ priori what influence the vibrations of the bow have on the clearness of the vibrations of the string, and consequently on the purity of the sounds produced. This influence is probably not to be neglected. It is known that a double-base, a tenor, or a violin bow cannot be employed indifferently for an instrument; it is also known that artists bestow great care in the choice which they make among bows of the same kind, and that they adopt certain tensions for the hairs. It would be interesting to submit the properties of bows to experimental analysis, and perhaps some result useful to practical music might be found. When the tension of the bow is such, that the sound of each hair has for octave of higher or lower order the sound of the vibrating string, or one of its consonances, would the sound produced be finer than under other conditions?

The analytical solution of the problem of vibrating strings is remarkably beautiful, and yet it is incomplete. To explain the effects produced, it is not enough, in practice, to recur, as is clone in analysis, to very various primitive states. Ordinarily the initial state is the state of equilibrium under the influence of the tension of the string, and yet what variety of effects does the bow produce!

Ordinary analysis does not even suffice in the very simple 
case in which the string is pinched. The initial figure and the primitive velocities are not arbitrarily given; they depend on the shock, and can be known only when the shock is exactly known.

The difficulty presented by the analytical theory of the bow, and even of the isolated shocks produced by pinching a string, is in fact that general and hitherto insurmountable difficulty, of subjecting the phænomenon of the shock to an exact analysis. It is known that only one very particular case of this phænomenon has been able to be approached, that only which Poisson has developed.

M. Duhamel does not consider the action of the bow under the point of view which has just been indicated. He regards the friction of the bow as equivalent, not to a series of shocks, but to a system of constant forces. A centre of attraction placed beside the string, far enough off for the displacements to be comparatively imperceptible, might therefore, according to this view, be a substitute for the bow. This seems at first sight scarcely probable. Be it as it may, if the new theory be exact, it should explain all the effects of the bow, and moreover its consequences should agree with the results of experiment.

M. Duhamel cites an experiment of verification, fiom which he has found that a circular bow, or ratber a friction-wheel acting upon a stretched string, causes it to deviate from its position of equilibrium, and brings it in a very short time to a rew position of equilibrium in which the friction maintains it, withont the string continuing to produce a sound. This fact, the result of M. Duhamel's theory and experiments, may be regarded as negative with relation to this very positive fact, that burdy-gurdy players sustain the sound of a string for whole hours, if desired. If $\mathbf{M}$. Duhamel has obtained a negative result, this is caused probably by his not having placed the wheel in the conditions in which it acts in the manner of the bow.

In fine, it is not easy to see how M. Duhamel's theory accounts for the four principal effects of the bow, and how it has reference to the vibrations which the hairs of the bow constantly execute when they are passed over a sonorous string. 